\title{
DETERMINAÇÃO DOS LIMITES DE DETECÇÃO (LD) E QUANTIFICAÇÃO (LQ) EM ANÁLISE DE RESÍDUOS DE PESTICIDAS ORGANOHALOGENADOS POR CROMATOGRAFIA EM FASE GASOSA
}

\begin{abstract}
Os limites de detecção (LD) e de quantificação (LQ) de métodos para análise de resíduos de pesticidas em determinada matriz são essenciais para o estabelecimento da capacidade analítica na determinação qualitativa e quantitativa de traços de substâncias químicas embutidos na mesma. A determinação desses limites em uma matriz exige a condução de ensaios experimentais com a proposição dos LQ e LD em níveis de concentrações que atinjam a sensibilidade a que o método se propõe. Em análise de resíduos de pesticidas em alimentos, os Limites Máximos de Resíduos (LMR) permitidos para cada tipo de cultura estabelecidos pela ANVISA (mg/kg de peso da matriz) podem constituir parâmetro inicial. Neste estudo, apresentou-se metodologia para a determinação desses limites em maçã para os seguintes pesticidas organohalogenados: clorotalonil, captana, dicofol, endossulfam I, endossulfan II, endossulfan sulfato e vinclozolina. Os resultados de LD e LQ do método ensaiado para a exatidão $(\mathrm{RM} \%=64 \%$ a $127 \%)$ e precisão $(C V \%=2,11 \%$ a $12,55 \%)$ enquadraram-se nos valores indicados na literatura para considerar tais parâmetros como validados. Além disso, a metodologia empregada demonstrou capacidade para atingir valores de LQ e LD em concentrações abaixo dos recomendados pela legislação com a utilização de técnicas analíticas alternativas (CG/ECD/ECD) para a confirmação dos resultados.
\end{abstract}

PALAVRAS-CHAVE: LIMITE DE DETECÇÃO; LIMITE DE QUANTIFICAÇÃO; PESTICIDAS.

* Mestre em Tecnologia de Alimentos, Universidade Federal do Paraná (UFPR), Laboratório Central do Estado, Curitiba, PR (e-mail: marvinaimoto@sesa.pr.gov.br).

** Doutor em Química, Professor, Programa de Pós-Graduação em Tecnologia de Alimentos, UFPR, Curitiba, PR (e-mail: rfreitas@brturbo.com.br). 


\section{INTRODUÇÃO}

Em análise de resíduos de pesticidas, a determinação do limite de detecção do método (LD) representa a mais baixa concentração do analito em exame na relação ingrediente ativo: matriz que pode ser detectada com certa confiabilidade utilizando determinado procedimento experimental. Já a determinação do limite de quantificação (LQ) representa a mais baixa concentração que pode ser identificada e quantificada em determinada matriz com certo limite de confiabilidade, geralmente entre $95 \%$ e $99 \%$. As duas determinações correspondem a testes limites que especificam se os pesticidas em exame estão ou não acima ou abaixo de determinado valor (expresso em $\mathrm{mg} / \mathrm{kg}$ da amostra), sendo que a precisão e a exatidão das determinações também devem ser registradas para que o método seja validado (SCHARTZ e KRULL, 1998).

Para a validação dos níveis dos limites de detecção (LD) e de quantificação (LQ) de qualquer pesticida, certo número de amostras fortificadas com os compostos de interesse deve ser analisado próximo ao nível de concentração desejado (geralmente próximo ao menor ponto da curva de calibração) em que será possível detectar e/ou quantificar os analitos (THIER e ZEUMER, 1987; EUROPEAN COMISSION, 2000a). Para a determinação dos valores da concentração dos limites de deteç̧ão (LD) e quantificação (LQ) a serem analisados, as concentrações dos pesticidas são calculadas como se estivessem em determinada amostra (expressas em mg de pesticida por $\mathrm{kg}$ da amostra), estabelecendose os valores por convenções descritas na literatura (THIER e ZEUMER, 1987; AOAC, 1993; ICH, 1996; RIBEIRO, 1997; CURRIE, 1999; EUROPEAN COMISSION, 2000a; ABNT, 2001; GARP, 2001; BRITO et al., 2002).

A ICH (1996) e SCHARTZ e KRULL (1998) reconhecem a convenção sinal: ruído 3:1 e 10:1 para as determinações dos LD e LQ, respectivamente, utilizada para as técnicas cromatográficas que apresentam a linha de base do solvente. Também indicam as seguintes alternativas para a determinação dos LD e LQ: métodos visuais não-instrumentais e média dos LD e LQ calculados. Nos primeiros métodos podem ser incluídos os LD e LQ determinados por técnicas cromatográficas em camada delgada (CCD) ou titulação. Os LD e LQ também podem ser calculados com base no desvio-padrão (DP) da resposta e a inclinação ( 1 ) da curva de calibração (média de 3 curvas), obtidos em ensaios de recuperação em níveis próximos ao LD e LQ a partir das equações 1 e 2:

Em que:

$$
\begin{array}{ll}
\mathrm{LD}=3,3 \mathrm{DP} / \mathrm{I} & \text { (equação 1) } \\
\mathrm{LQ}=10 \mathrm{DP} / \mathrm{I} & \text { (equação 2) }
\end{array}
$$

DP = estimativa do desvio-padrão da resposta ou do branco instrumental (3 curvas);

I = Inclinação ou coeficiente angular da curva analítica.

A estimativa dos limites por meio da curva é em geral utilizada para análise de soluçõespadrão e está relacionada com ruídos instrumentais. Em alguns métodos, o valor do sinal e o desviopadrão podem variar bastante para a amostra em branco e a amostra testemunha que apresenta muitos interferentes.

Com o objetivo de demonstrar a determinação dos LD e LQ de alguns pesticidas organohalogenados para processos de validação em uma matriz utilizou-se o método de sinal: ruído e escolheu-se o detector de captura de elétrons (ECD) pela sua maior sensibilidade frente a esses tipos de compostos.

\section{MATERIAL E MÉTODOS}

Maçãs orgânicas, previamente analisadas e isentas de qualquer tipo de resíduo químico que 
pudesse interferir nas análises, foram utilizadas como matriz para os ensaios da determinação dos limites de detecção LD) e quantificação (LQ) neste estudo.

\subsection{SISTEMA DE CROMATOGRAFIA EM FASE GASOSA (CG/ECD/ECD)}

O sistema cromatográfico utilizado para a condução dos ensaios consistiu de cromatógrafo a gás, marca Varian (modelo CP 3800), equipado com amostrador automático, acoplado simultaneamente a duas colunas de diferentes polaridades para confirmação dos resultados e dois detectores de captura de elétrons (CG/ECD/ECD), construidos com fonte radioativa de $\mathrm{Ni}_{63}$, sensível a compostos organohalogenados. As colunas utilizadas foram: CP Sil-8 de sílica fundida, marca Chrompack, com 95\% de dimetilpolissiloxano e 5\% de fenil-dimetilpolissiloxano (30 m de comprimento $\times 0,32 \mathrm{~mm}$ de diâmetro x 0,25 $\mu \mathrm{m}$ de espessura do filme) e CP Sil-9 de sílica fundida, marca Chrompack, com 50\% de dimetilpolissiloxano e $50 \%$ de fenil-dimetilpolissiloxano $(30 \mathrm{~m}$ de comprimento $\times 0,32 \mathrm{~mm}$ de diâmetro $\times 0,25 \mu \mathrm{m}$ de espessura do filme).

As condições cromatográficas empregadas foram: rampa $-100^{\circ} \mathrm{C}$ por $3 \mathrm{~min}$; de $100^{\circ} \mathrm{C}$ a $180^{\circ} \mathrm{C}$ por $10^{\circ} \mathrm{C} / \mathrm{min}$; de $180^{\circ} \mathrm{C}$ a $250^{\circ} \mathrm{C}$ por $2^{\circ} \mathrm{C} / \mathrm{min}$, por $5 \mathrm{~min}=51 \mathrm{~min}$. Temperatura do detector de $300^{\circ} \mathrm{C} \mathrm{e}$ temperatura do injetor de $240^{\circ} \mathrm{C}$. Usou-se como gás de arraste Nitrogênio 5,0 analítico para $E C D$, com fluxo de $1,5 \mathrm{~mL} / \mathrm{min}$ e modo de injeção splitless. O volume da amostra injetado no cromatógrafo foi de $1 \mu \mathrm{L}$.

\subsection{COMPOSTOS ORGANOHALOGENADOS}

Os compostos organohalogenados para a determinação dos limites de detecção (LD) e quantificação (LQ) na matriz selecionada foram: clorotalonil, captana, dicofol, endossulfam I, endossulfam II, endossulfam sulfato e vinclozolina. As estruturas químicas dos compostos estão demonstradas na Figura 1.

Para o estabelecimento das concentrações a serem ensaiadas foram injetadas misturas das soluções dos pesticidas em concentrações mínimas e conhecidas até a obtenção de cromatogramas com picos que resultassem na razão sinal:ruído 3:1 e 10:1 para os limites de detecção e quantificação, respectivamente (KEITH et al., 1983; RIBEIRO, 1997; CURRIE, 1999; EUROPEAN COMISSION, 2000a).

\subsection{FORTIFICAÇÃO E EXTRAÇÃO DOS PESTICIDAS \\ ORGANOHALOGENADOS}

Com base nos valores das concentrações dos pesticidas que resultaram em melhores cromatogramas (relação sinal/ruído 10:1) estipulou-se a concentração dos pesticidas em $1 \mathrm{LQ}$. Essa concentração foi proposta nos ensaios a serem conduzidos para verificação da recuperação dos pesticidas na matriz em níveis que satisfizessem os parâmetros de precisão e exatidão necessários (KEITH et al., 1983; ICH, 1996; RIBEIRO, 1997).

Para a condução dos ensaios foram fortificadas 5 replicatas de cada pesticida com a mistura das soluções-padrão nas concentrações propostas dos LQ mais 2 replicatas da testemunha (branco + maçã), segundo recomendações do Guia para análise de pesticidas (EUROPEAN COMMISSION, 2000a). Para correção do efeito da matriz na linha de base dos cromatogramas, as fortificações com a mistura dos padrões foram feitas com a solução obtida pelo mesmo processo de extração da testemunha, bem como a construção das curvas analíticas. Para a União Européia, o quantitativo do efeito da matriz na linha de base não deve ultrapassar 30\% do LQ dos pesticidas (EUROPEAN COMMISSION, 2000a).

Para a extração dos pesticidas organohalogenados empregou-se o método multi-resíduo, modelo europeu (ZOONEN et al., 1996), utilizando $30 \mathrm{~mL}$ de acetona e $60 \mathrm{~mL}$ da mistura de diclorometano e n-hexano (30:30). 


\section{FIGURA 1 - ESTRUTURA QUÍMICA DOS COMPOSTOS ORGANOHALOGENADOS}<smiles>N#Cc1c(Cl)c(Cl)c(Cl)c(C#N)c1Cl</smiles>

Tetrachloroisophtalonitrile

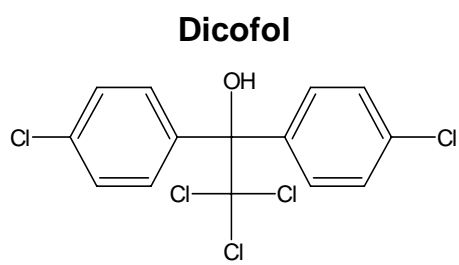

2,2,2-thricloro-1,1-bis(4-chlorophenyl)
ethanol<smiles>O=C1CCCCCN1SN1C(=O)C2CC=CCC2C1=O</smiles>

$\mathrm{N}$ - (trichoromethylthio)cyclohex-4-ene-

\section{1,2-dicarboximide}

Endossulfam I, II e sulfato<smiles>O=C1OCC23COCOC2C(Cl)(C(Cl)=C3Cl)C1(Cl)Cl</smiles>

(1,4,5,6,7-7-hexachloro-8,9,10-trinorborn5-em,2,3-ylenebismethylene) sulfite

Vinclozolina<smiles>C=CC1(C)OC(=O)N(c2cc(Cl)cc(Cl)c2)C1=O</smiles>

(RS) - 3 - (3,5 dichlorophenyl) -5-methyl -5 - vinyl - 1,3 - oxazolidine - 2,4 - dione

Nota: Nomenclatura química segundo a IUPAC.

\subsection{DETERMINAÇÃO DO LQ ESTATÍSTICO (LQE)}

Após o processo de recuperação dos pesticidas pelo método de escolha calculou-se a média das recuperações $(x)$, o desvio-padrão $(s)$ das quantidades recuperadas e o valor do LQ estatístico (LQE), definido como $10 \times$ s. Considerou-se o LQ proposto aceitável quando o valor foi maior ou igual ao LQ estatístico (LQE) encontrado nos ensaios (KEITH et al., 1983).

Avaliou-se a exatidão dos resultados pela média das recuperações (RM\%) para verificar sua proximidade com o valor real (AOAC, 1993), que dependendo da proximidade da concentração do menor ponto da curva de calibração, as recuperações podem variar entre 60\% e 140\% (EUROPEAN COMISSION, 2000a) e de $50 \%$ a $150 \%$ (USDA, 2002).

Os resultados obtidos foram submetidos ao teste de hipóteses, sendo estabelecida como hipótese nula $\left(H_{0}\right)=$ Rec. igual a 100\% e como hipótese alternativa $\left(H_{1}\right)=$ Rec. diferente de 100\% (BRITO et al., 2003). Os valores das recuperações foram submetidos a tratamento estatístico pelo teste de Grubbs para identificação de possíveis outliers, sendo permitido no máximo 1 outlier para cada nível de fortificação no método para validação dos parâmetros (EUROPEAN COMISSION, 2000a).

Para avaliar a precisão foram calculados os coeficientes de variação relativos (CV\%), que 
segundo a literatura e os níveis de concentração ensaiados devem ser inferiores a 20\% (THIER e ZEUMER, 1987) e as variações compreendidas entre 16\% a 23\% (HARRIS, 2001; LEITE, 2002). As equações 3 e 4 foram utilizadas para obtenção da RM\% e do CV\%:

$$
\begin{aligned}
& \mathrm{RM} \%=\frac{\mathrm{R} 1+\mathrm{R} 2+\mathrm{R} 3+\mathrm{R} 4+\mathrm{R} 5}{\mathrm{n}} \times 100 \quad \text { (equação 3) } \\
& \mathrm{CV} \%=\frac{\mathrm{s}}{\mathrm{RM}} \quad \text { (equação 4) }
\end{aligned}
$$

Em que:

$\mathrm{R} 1+\ldots \mathrm{R} 5$ = soma das recuperações obtidas nas cinco replicatas;

$\mathrm{RM}=$ média das recuperações;

$s=$ desvio-padrão das recuperações;

$\mathrm{n}=$ número de replicatas ensaiadas.

Para a determinação média dos limites de detecção e de quantificação do método foram aplicadas as equações 5 e 6 ( THIER et al., 1987):

$$
\begin{array}{r}
\mathrm{LDM}=\frac{\text { concentração do LQ }}{3 \mathrm{DP}} \\
\mathrm{RM}
\end{array} \quad \begin{aligned}
& \text { (equação 5) } \\
& \mathrm{LQM}=\underline{\text { concentração do LQ }} \times 10 \mathrm{DP} \quad \text { (equação 6) } \\
& \mathrm{RM}
\end{aligned}
$$

Em que:

Concentração do LQ = concentração do LQ proposto para cada pesticida;

$\mathrm{RM}=$ médias das cinco recuperações;

DP = desvio-padrão obtido das cinco recuperações.

\section{RESULTADOS E DISCUSSÃO}

$\mathrm{Na}$ Tabela 1 constam as concentrações dos pesticidas encontradas nos cromatogramas com a relação sinal/ruído 10:1 para os valores dos limites de quantificação (1LQ) propostos.

\section{TABELA 1 - CONCENTRAÇÕES DOS PESTICIDAS PROPOSTAS PARA A DETERMINAÇÃO DO LQ}

\begin{tabular}{ccc}
\hline Pesticida & $\begin{array}{c}\text { Nível de concentração } \\
(\mathbf{m g} / \mathbf{K g})\end{array}$ & $\begin{array}{c}\text { LQ proposto } \\
(\mathbf{m g} / \mathbf{K g})\end{array}$ \\
\hline Clorotalonil & 0,02 & $1 \mathrm{LQ}$ \\
\hline Captana & 0,1 & $1 \mathrm{LQ}$ \\
\hline Dicofol & 0,1 & $1 \mathrm{LQ}$ \\
\hline Endossulfam I & 0,02 & $1 \mathrm{LQ}$ \\
\hline Endossulfam II & 0,02 & $1 \mathrm{LQ}$ \\
\hline Endossulfam sulfato & 0,02 & $1 \mathrm{LQ}$ \\
\hline Vinclozolina & 0,02 & $1 \mathrm{LQ}$ \\
\hline
\end{tabular}


As Figuras 2 e 3 mostram os cromatogramas obtidos com a amostra testemunha e os sinais dos picos com a razão 10:1 para a determinação das concentrações dos pesticidas para o nível de 1 LQ proposto.

FIGURA 2 - CROMATOGRAMA DA TESTEMUNHA

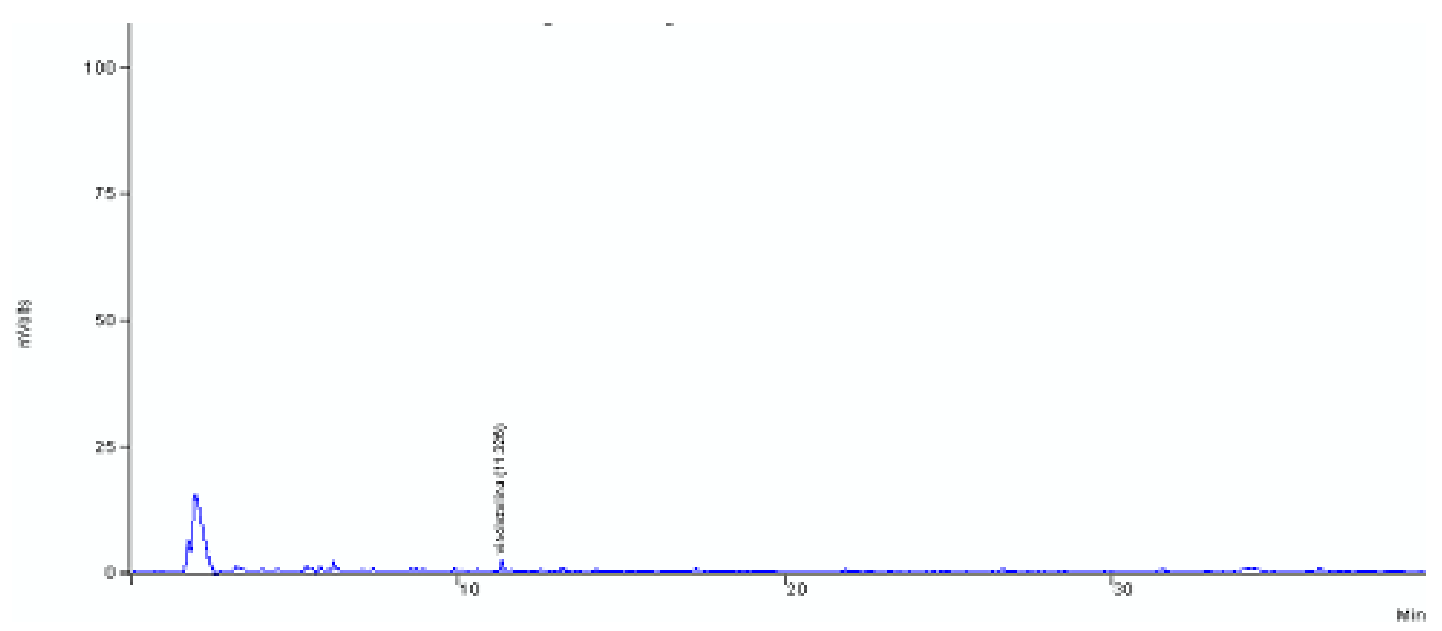

FIGURA 3 - CROMATOGRAMA DA MISTURA DOS PESTICIDAS

COM O NÍVEL 1 LQ (10:1)

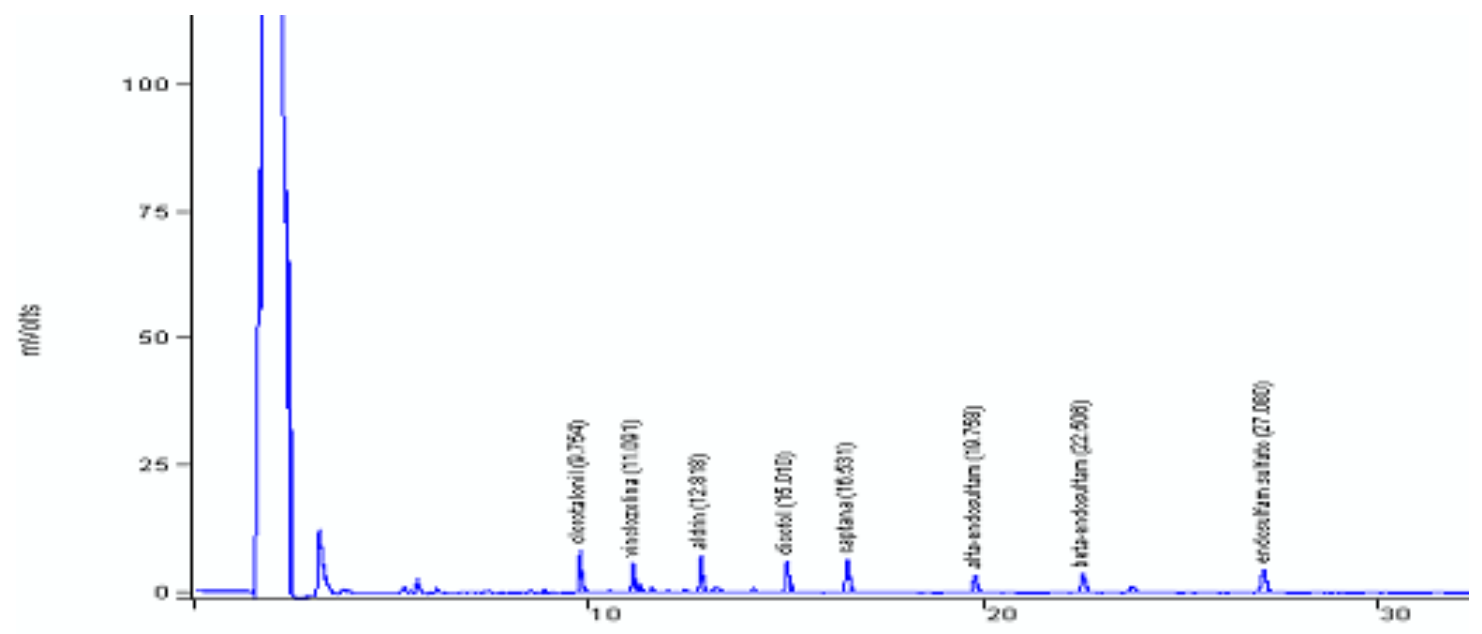

O cromatograma da testemunha (Figura 2) apresentou um pico no tempo de retenção do pesticida vinclozolina, tendo a sua integração anulada pela quantificação dos pesticidas na curva de calibração construída no extrato orgânico da testemunha.

A Tabela 2 demonstra as recuperações obtidas nas cinco replicatas no nível 1 LQ proposto com o valor calculado para o LQ estatístico (LQE), bem como o percentual médio das recuperações obtidas (RM\%). 


\section{TABELA 2 - DEMONSTRATIVO DAS RECUPERAÇÕES, RM, s, LQE ESTATÍSTICO E \% MÉDIA DAS RECUPERAÇÕES (\%RM)}

\begin{tabular}{ccccccccccc}
\hline Pesticida & $\begin{array}{c}\text { Nivel } \\
\text { (mg/kg) }\end{array}$ & R1 & R2 & R3 & R4 & R5 & RM & s & $\begin{array}{c}\text { LQE } \\
\text { estatístico }\end{array}$ & $\begin{array}{c}\text { RM } \\
\%\end{array}$ \\
\hline Clorotalonil & 0,02 & 0,019 & 0,021 & 0,022 & 0,022 & 0,021 & 0,021 & 0,0012 & $1,2 \mathrm{E}-3$ & 107 \\
\hline Captana & 0,1 & 0,114 & 0,129 & 0,123 & 0,129 & 0,125 & 0,124 & 0,0061 & $6,1 \mathrm{E}-3$ & 127 \\
\hline Dicofol & 0,1 & 0,087 & 0,078 & 0,093 & 0,089 & 0,095 & 0,088 & 0,0066 & $6,6 \mathrm{E}-3$ & 88 \\
\hline Endossulfam I & 0,02 & 0,012 & 0,012 & 0,013 & 0,012 & 0,013 & 0,012 & 0,00043 & $4,0 \mathrm{E}-3$ & 64 \\
\hline Endossulfam II & 0,02 & 0,014 & 0,013 & 0,014 & 0,017 & 0,017 & 0,015 & 0,0019 & $1,9 \mathrm{E}-3$ & 76 \\
\hline Endossulfam & 0,02 & 0,022 & 0,022 & 0,020 & 0,021 & 0,021 & 0,021 & 0,00067 & $6,0 \mathrm{E}-4$ & 108 \\
sulfato & & & & & & & & & & \\
\hline Vinclozolina & 0,02 & 0,013 & 0,014 & 0,014 & 0,014 & 0,014 & 0,014 & 0,0003 & $3,0 \mathrm{E}-4$ & 70 \\
\hline
\end{tabular}

R1,R2,R3,R4,R5 = recuperações obtidas nas análises; RM = média das recuperações; $s$ = desvio-padrão; LQE estatistico $\mathrm{e}$ $\% \mathrm{RM}=$ percentual médio das recuperações.

Como todos os valores de LQ estatístico (LQE) de cada pesticida ensaiado foram menores que os LQ propostos inicialmente, esses poderão ser assumidos conforme a condição pré-estabelecida na metodologia utilizada (KEITH et al., 1983). A média das recuperações dos pesticidas obtidas, entre 64\% a 127\%, também mostra a aceitabilidade da performance analítica do método multi-resíduo utilizado nos níveis de concentração ensaiados dos pesticidas, pois a literatura da área estabelece recuperações entre $50 \%$ a 150\% (USDA, 2002) e 40\% a 160\% (EUROPEAN COMMISSION, 2000b).

A Tabela 3 indica os limites de detecção (LD) e os limites de quantificação (LQ) encontrados para os pesticidas ensaiados em maçã, além dos Limites Máximos de Resíduos (LMR) permitidos pela legislação em vigor (ANVISA, 2007).

TABELA 3 - LIMITES DE DETECÇÃO (LD), LIMITES DE QUANTIFICAÇÃO (LQ), LMR $^{1}$ DOS PESTICIDAS ORGANOHALOGENADOS EM MAÇÃ

\begin{tabular}{|c|c|c|c|}
\hline Pesticidas & $\begin{array}{l}\text { Limite de detecção } \\
\qquad(\mathrm{mg} / \mathrm{kg})\end{array}$ & $\begin{array}{l}\text { Limite de quantificação } \\
\qquad(\mathrm{mg} / \mathrm{kg})\end{array}$ & $\begin{array}{l}\mathrm{LMR}^{1} \\
(\mathrm{mg} / \mathrm{kg})\end{array}$ \\
\hline Clorotalonil & 0,005 & $0,00123 \therefore 0,01$ & 1,0 \\
\hline Captana & 0,03 & $0,0061 \therefore 0,05$ & 25 \\
\hline Dicofol & 0,03 & $0,0066 \therefore 0,05$ & 5,0 \\
\hline Endossulfam I & 0,01 & $0,004 \therefore 0,02$ & NA \\
\hline Endossulfam II & 0,005 & $0,0019 \therefore 0,02$ & NA \\
\hline Endossulfam sulfato & 0,005 & $0,0006 \therefore 0,01$ & NA \\
\hline Vinclozolina & 0,005 & $0,0003 \therefore 0,01$ & NA \\
\hline
\end{tabular}

$\mathrm{LMR}^{1}=$ Limites máximos de resíduos (ANVISA, 2007).

Os valores encontrados para LD e LQ foram arredondados para a $3^{\mathrm{a}}$ e $2^{\mathrm{a}}$ casas após a vírgula para obtenção de resultados com significado toxicológico (LEITE, 2002), bem como para o cumprimento da legislação (ANVISA, 2007).

Na Tabela 4 constam os resultados obtidos para a exatidão e precisão dos ensaios. 
TABELA 4 - DEMONSTRATIVO DA EXATIDÃO E PRECISÃO DOS ENSAIOS

\begin{tabular}{ccccccc}
\hline Pesticida & $\begin{array}{c}\text { Nivel } \\
(\mathbf{m g} / \mathbf{k g})\end{array}$ & $\begin{array}{c}\text { RM } \\
(\mathbf{m g} / \mathbf{k g})\end{array}$ & $\mathbf{n}$ & $\mathbf{t}_{\text {calculaco }}$ & $\mathbf{s}$ & $\begin{array}{c}\text { CV } \\
\%\end{array}$ \\
\hline Clorotalonil & 0,02 & 0,0215 & 5 & 2,4590 & $1,22 \mathrm{E}-4$ & 5,66 \\
\hline Captana & 0,1 & 0,124 & 5 & 7,7922 & $6,16 \mathrm{E}-4$ & 4,97 \\
\hline Dicofol & 0,1 & 0,0884 & 5 & $-3,5151$ & $6,6 \mathrm{E}-4$ & 7,48 \\
\hline Endossulfam I & 0,02 & 0,0127 & 5 & $-33,953$ & $4,3 \mathrm{E}-5$ & 3,41 \\
\hline Endossulfam II & 0,02 & 0,0152 & 5 & $-5,0526$ & $1,9 \mathrm{E}-4$ & 12,55 \\
\hline Endossulfam sulfato & 0,02 & 0,0215 & 5 & 2,4590 & $6,7 \mathrm{E}-5$ & 5,66 \\
\hline Vinclozolina & 0,02 & 0,0140 & 5 & $-41,1034$ & $2,9 \mathrm{E}-5$ & 2,11 \\
\hline
\end{tabular}

$\mathrm{RM}=$ Recuperação média; $\mathrm{n}=$ número de amostras analisadas; $\mathrm{t}_{\text {tabelado }} 95 \%=2,776 ; \mathrm{S}=$ desvio-padrão; $\mathrm{CV} \%=$ coeficiente de variação.

O teste $t$ de Student aplicado mostrou a inexatidão da recuperação média obtida para o pesticida captana em que o valor do $t_{\text {calculado }}$ foi superior ao $t_{\text {tabelado }}$ para o intervalo de confiança de $95 \%$ $(2,776)$ e 5 graus de liberdade. No entanto, isso não representou diferença significativa nos níveis de concentração ensaiados, uma vez que a sensibilidade do método atendeu aos critérios demonstrados na Tabela 5 (EUROPEAN COMMISSION, 2000a).

\section{TABELA 5 - CRITÉRIOS PARA ESTABELECIMENTO DO LQ SEGUNDO A EUROPEAN COMMISSION, 2000a}

\begin{tabular}{c|c}
\hline LMR ( $\mathbf{m g} / \mathbf{k g})$ & *LQ $\mathbf{( m g / k g )}$ \\
\hline$>0,1$ & $\leq 0,1$ \\
\hline 0,1 & $\leq 0,05$ \\
\hline 0,05 & $\leq 0,02$ \\
\hline$<0,05$ & $\leq \mathrm{LMR} \times 0,5$
\end{tabular}

LMR = Limite Máximo Resíduo (EUROPEAN COMMISSION, 2000a); *LQ (mg/kg) = exceto quando o valor proposto for o LMR $(\mathrm{mg} / \mathrm{kg})$.

Pela Tabela 5, e segundo os critérios da comunidade européia, os valores de LQ encontrados para os pesticidas clorotalonil $=0,01$ e dicofol $=0,05$ foram ainda menores que os exigidos, os quais poderiam ser $\leq 0,1 \mathrm{mg} / \mathrm{kg}$ para $L M R>0,1$. Como foram encontrados valores de $L Q$ menores que os propostos, esses resultados também poderão ser assumidos se após reaplicação dos mesmos critérios se obtiver a exatidão e a precisão necessárias para aceitação pela literatura como validados.

A determinação da precisão do método foi avaliada pelo cálculo dos coeficientes de variação relativos das recuperações (CV\%), e os resultados enquadraram-se na faixa aceitável para os níveis de concentração trabalhados segundo dados da literatura (THIER e ZEUMER, 1987; ZOONEN et al., 1996; HARRIS, 2001; LEITE, 2002, CODEXALIMENTARIUS COMMISSION, 2004). Entretanto, algumas considerações devem ser efetuadas para diminuir as maiores fontes de incerteza de medição do método. Um dos fatores mais importante nesse tipo de análise em que são injetadas amostras consideradas "sujas", uma vez que não são realizadas as etapas de purificação das amostras antes das injeções, é o chamado efeito matriz. Devido à complexidade do binômio principio ativo / matriz, os co-extrativos da amostra podem vir a interagir no sistema cromatográfico alterando a resposta do 
equipamento e provocando a super estimação dos resultados. Esse fenômeno (carry over) se deve possivelmente à impregnação dos co-extrativos da matriz nos sítios ativos da coluna e/ou liners, preenchendo todo o espaço que seria ocupado pelos radicais livres da substância química em exame. Assim, devido às sucessivas injeções, a quantidade do analito a ser determinada passa a ser cada vez maior. Para a correção e prevenção do efeito matriz deve-se utilizar testemunhas (branco + matriz) no método e na construção das curvas analíticas para as determinações dos quantitativos. Assídua manutenção do sistema cromatográfico (troca de septos e liners, verificação do tempo de uso das colunas, lâmpadas dos detectores, qualidade dos gases, etc.) deve ser rigorosamente observada para a obtenção de constante resposta do equipamento e assim maior confiabilidade nas medidas realizadas.

Cabe ressaltar que com a utilização de equipamentos e detectores de maior complexidade (GC/MS e GC/MS/MS) como técnicas de confirmação serão determinados níveis de LD e LQ cada vez menores, aumentando a capacidade analítica, a sensibilidade e a especificidade desse tipo de análise.

\title{
4 CONCLUSÃO
}

Como todos os valores dos LQ estatísticos (LQE) resultaram abaixo dos valores de LQ propostos, e de acordo com a condição inicial pré-estabelecida, esses foram assumidos para todos os pesticidas ensaiados segundo a metodologia utilizada. Os valores obtidos para a determinação dos limites de detecção (LD) e de quantificação (LQ) mostraram-se satisfatórios, uma vez que os resultados encontrados para a exatidão e precisão do método multi-resíduo empregado enquadraram-se no propósito de validação desses parâmetros no método para análise dos pesticidas na maçã.

\begin{abstract}
ESTABLISHMENT OF DETECTION AND QUANTITATION LIMITS IN ANALYSIS OF ORGANOHALOGENATE PESTICIDES RESIDUES BY GAS CHROMATOGRAPHY

The detection (DL) and quantification limits (QL) of methods for pesticide residues analysis in a determined matrix are essential for the establishment of analytical capacity in qualitative and quantitative determination of traces of chemicals substances inherent. The determination of these limits in a matrix requires conducing experimental assays using concentration levels of DL and QL that reach the sensibility of the method. In pesticide residues analysis in food, the maximum residues limits (LMR) established for each culture by ANVISA (mg/kg of matrix weight), can constitute an initial parameter. In this study, a methodology to determinate these limits in apple were presented for the following organohalogenate pesticides: Chlorothalonil, Captan, Dicofol, Endosulfan I, Endosulfan II, Endosulfan sulfate and Vinclozolin. The results of DL and QL of the used method for accuracy $(\mathrm{RM} \%=64 \%$ to $127 \%)$ and precision $(\mathrm{CV} \%=2.11 \%$ to $12.55 \%)$, are within the values found in literature to consider such parameters as validated. Besides, the used methodology demonstrated capacity to reach values of QL and DL in concentrations below the recommended by brazilian legislation with the utilization of alternative analytical techniques (GC/ECD/ECD) to confirm the results.
\end{abstract}

KEY-WORDS: LIMITS OF DETECTION; LIMITS OF QUANTIFICATION; PESTICIDES.

\section{REFERÊNCIAS}

1 ANVISA. Agência Nacional de Vigilância Sanitária. Monografias. Disponível em: http:// www.anvisa.gov.brl toxicologia/monografias.pdf. Acesso em: 13/12/2007.

2 ABNT. Associação Brasileira de Normas Técnicas. NBR ISO/IEC 17025: requisitos gerais para competência de laboratórios de ensaio e calibração. Rio de Janeiro, 2001. 20 p.

3 AOAC. Association of Official Analytical Chemists. Peer verified method program: manual on policies and procedures. Arlington, VA, 1993.

4 BRITO, N. M.; AMARANTE JR, O. P.; POLESE, L.; SANTOS, T.C.R.; RIBEIRO, M. L. Avaliação da exatidão e da precisão de métodos de análise de resíduos de pesticidas mediante ensaios de recuperação. Pesticidas: r. ecotoxicol. e meio ambiente, Curitiba, v.12, p. 155-166, jan./dez. 2002. 
5 BRITO, N. M.; AMARANTE JR, O. P.; POLESE, L.; RIBEIRO, M. L. Validação de métodos analíticos: estratégia e discussão. Pesticidas: r. ecotoxicol. e meio ambiente, Curitiba, v.13, p. 129-146, jan./dez. 2003.

6 CODEX ALIMENTARIUS COMMISSION. Report of the working group on methods of analysis and sampling to the $\mathbf{3 6}^{\text {th }}$ session of the CCPR. New Delhi, April, 2004. (Documento CRD 5).

7 CURRIE, L. A. Detection and quantification limits: origins and historical overview. Anal. Chem. Acta, v. 391, p.127 134, 1999.

8 EUROPEAN COMMISSION. Directorate General Health and Consumer Protection. Guidance document on residue analytical methods. North Yorkshire, 2000a. 16 p. (Documento SANCO /825/00, rev. 6).

9 EUROPEAN COMMISSION. Directorate General Health and Consumer Protection. Quality control procedures for pesticide residue analysis. North Yorkshire, 2000b. 30 p. (Documento SANCO /3103/00, second edition).

10 GARP. Associação Grupo de Analistas de Resíduos de Pesticidas. Validação de métodos: avaliação de conformidade. São Paulo, 2001.

11 HARRIS, D. C. Análise química quantitativa. 5. ed. Rio de Janeiro: LTC Editora, 2001.

$12 \mathrm{ICH}$. International Conference on Harmonization. Validation of analytical procedures: methodology. London, 1996. 9 p. (ICH Harmonized Tripartide Guideline CPMP/ICH/281/95).

13 KEITH, L. H. et al. Principles of environmental analysis. Anal. Chem., v. 55, p. $2210-18,1983$.

14 LEITE, F. Validação em análise química. 4. ed. Campinas, SP: Editora Átomo, 2002.

15 RIBEIRO, M. L. Validação de metodologia analítica visando a determinação de resíduos de pesticidas. In: ENCONTRO NACIONAL DE ANALISTAS DE PESTICIDAS, 19., São Paulo, 1997. Anais... São Paulo: GARP/IAL, 1997. p. 89 -95.

16 SCWARTZ, M. E.; KRULL, I. S. Validação de métodos cromatográficos. Pharmaceutical Technology. v. 2, n.3, p. $12-20,1998$

17 THIER, H. P.; ZEUMER, H. Manual of pesticide analysis. New York: Verlag Chemie, 1987. p. 37-41.

18 USDA. United State Department of Agriculture. Pesticide Date Program. Acceptability criteria for process control and fortification recoveries. Washington, DC., 2002. 8 p. (SOP- No.: PDP -QC-04, Revision 7).

19 ZOONEN, P. et al. Analytical methods for pesticide residues in foodstuffs. Multi-residue method - Part 1 , Annex A -D. $6^{\text {th }}$ ed. Rijswijk: Inspectorate for Health Protection, 1996. 